http://jmscr.igmpublication.org/home/ ISSN (e)-2347-176x ISSN (p) 2455-0450

crossref DOI: https://dx.doi.org/10.18535/jmscr/v7i9.113

Journal Of Medical Science And Clinical Research

\title{
9 Years Retrospective Study with Movement Disorder on Syndopa \& Exercises - An Analysis of Factors Influencing Prognosis with Evidence
}

\author{
Author
}

\section{Dr S.S. Subramanian}

M.P.T (Orthopaedics), M.S (Education), M. Phil (Education), Ph.D (Physiotherapy)

The Principal, Sree Balaji College Of physiotherapy, Chennai - 100

Affiliated To (Bharath) University, BIHER, Chennai - 73, India

\begin{abstract}
Introduction: Movement disorder, a major component of Parkinson's disease can lead to dependency and disability. With an increased life expectancy health care of the ailments associated with elderly gets significant.

Aims \& Objectives: This original case study which aims at evaluating the clinical course and functional outcome along with factors influencing the prognosis on a subject with Parkinson's disease with 8 years follow up.

Materials and Methodology: This study was conducted during the period from February 2010 till April 2019. The subject was treated at his domiciliary with a set of exercises to both extremities and the spine, each session for 30-35 minutes with a frequency of thrice a week. Intensity of exercises were based on his energy levels, pain other physical and constitutional symptoms. The nature of exercise techniques included apart from passive stretching, Proprioceptive exercises using Physioball, balance training and walking re training. UPDRS was used once at the beginning in 2010 again in, 2014 and 2019, results were analyzed statistically

Results: UPDRS were in significant $P>.01$

Conclusion: impact of progressive nature of this chronic deteriorating condition requires not only adherence with physiotherapy but multiple parameters were influencing clinical prognosis of this condition with family support, genetic, individuals, attitude, perception and environment etc.

Keywords: $P D$ - Parkinson's disease, UPDRS, Proprioceptive Exercises.
\end{abstract}

\section{Introduction}

Parkinson disease (PD) is a complex neurodegenerative disorder, with wide reaching implications for patients and their families (Tomilson et al 2012). The management of PD has traditionally centered on drug treatment (Rascol etal 2000) but even with optimal medical management, patients still experience a deterioration of body function, daily activities, participation and decline in mobility (Scherag et al 2000). This can lead to increased dependence on others, in activity (Weintraub et al 1986). Social isolation resulting in reduced quality of life (Andrew etal 2013). Inclusion of rehabilitation therapies as an adjuvant to pharmacological and neurological treatment in the care of P.D towards multidisciplinary management are widely getting recognized (Gage \& Storey 2004) 
Physiotherapy for P.D focuses on transfers, posture, upper limb function, balance, gait physical capacity and activities. It also uses cueing strategies, cognitive movement strategies and exercise to maintain or increase independence, safety and quality of life (Kim et al 2013) owing to weak evidence base for people with P.D in physiotherapy. This original research with patient preferences and problem based therapy with evidence are attempted with an aim to enrich physiotherapy care for P.D on a longer duration follow up of 9 years

\section{Aims \& Objectives}

1. To analyse clinical course and functional activities in a nine year follow up of a Parkinson's disease subject

2. To evaluate the efficacy of physiotherapy on functional activities using UPDRS

3. To find out factors influencing prognosis among PD subjects

\section{Materials and Methodology \\ Background Information}

Retired from an insurance firm of India, 77 year old male, mesomorph with sedentary life style gives a medical

$\mathrm{H} / \mathrm{o}$

A hypertensive from 1990 on regular medication, was a known type II diabetic since 1993 has undergone coronary artery bye pass surgery in 1995, In 2010 had malena and his hemoglobin went down to $5 \mathrm{mg}$ was treated medically subsequently he gradually developed slowness of movements and on T.Syndopa 110mg since 2010 . (Complaints were) c/o difficulty in getting up from bed, walking and dependence on his daily activities.

O/E:

- Bed mobility he was partially dependant transfers from bed to chair, comfort, sofa, he was in need of physical assistance

- Left upper and lower extremities had hypertonicity and hyper reflexia
- Right knee osteoarthritic changes ,bilateral shoulders ranges beyond 100 restricted and painful, but elbow, wrist and grip bilaterally were good

- Forward head posture, flexed hips and knees, mobile thoracic kyphosis were recorded

- Painful, restriction of cervical lumbar, hips knees and ankle joint movements

- Ambulant with walking frame for short distance

- cognitive domain was good, but depression, indrawn, became an introvert with less communication

- Provisional diagnosis: left hemiparesis, dyskinesia, generalized geriatric postural defects.

\section{Treatment Used}

This original case study follow up with physiotherapeutic intervention was from 2010 till today. He was continuing medication as prescribed by his neuro physician, and his daily activities. After due consent and explanation, specific domiciliary physiotherapy were started this non pharmacological intervention with physical activities were applied with weekly thrice frequency each session lasts for 25-30 minutes.

UPDRS was used to evaluate his prognosis (which consisted of 14 items on 5 scale) once at the beginning of the study, after 4 years and after 9 years using motor examination component of UPDRS on a 5 point scale

\section{Table of results}

Table of results using paired ' $t$ ' test on UPDRS in 2010 (I), 2014 (II) and 2019 (III) of this PD subjects prognosis

\section{I \& II}

\begin{tabular}{|l|c|c|c|c|c|}
\hline & Mean & SD & SE & $\mathrm{t}$ & $\mathrm{p}$ \\
\hline Pre & 11 & 7 & 4.04 & -3.96 & \\
\hline Post & 27 & & & & $>.1$ \\
\hline
\end{tabular}

\section{II \& III}

\begin{tabular}{|l|c|c|c|c|c|}
\hline & Mean & SD & SE & t & p \\
\hline Pre & 27 & 6.76 & 3.90 & -4.10 & $>.1$ \\
\hline Post & 43 & & & & \\
\hline
\end{tabular}




\section{I \& III}

\begin{tabular}{|l|c|c|c|c|c|}
\hline & Mean & SD & SE & t & p \\
\hline Pre & 11 & 23.42 & 13.52 & -2.36 & $>.1$ \\
\hline Post & 43 & & & & \\
\hline
\end{tabular}

Kindly note $\mathrm{p}>.1$ it was insignificant statistically denoting negative prognosis

\section{Discussion}

\section{Years Clinical Prognosis of the Subject}

In 2010 he was ambulant unaided for short distance partially dependent for self-care. Social activities were less

In 2014, he has started using walker could manage independently dependency for self-care, has increased social activities have become rare

In 2017 requires assistance even with walker, level of dependency for self-care has increased a paid care giver was appointed round the clock, even bed mobility, transfers have become difficult, constitutional symptoms such as lack of appetite constipation, urinary in continence have increased

Occasions of long hours of sitting 4-6 hours was found to increase dyskinesia reaction time for physical activities a slow deterioration was noted

Physical functioning for daily activities dependency level was increasing gradually in the last 5 years cognitive domain was occasionally noted to be incoherent and irrelevant answers

Level of depression was noted to be increasing with lowering of self-care increasing dependency. Presently as on $30^{\text {th }}$ April 2019, the subject has developed hallucinations, withdrawing from socializing, needs support even for bed transfer and almost of his daily physical activities like eating, dressing, bathing, toileting and walking. Also physically dyskinesia has increased, with tremors and rigidity substantially become more restricting his mobility and leading to a total dependency level for all this physical daily activities.

\section{Critical apprisal of this presentation were}

1. Specific evaluatory scale were not used for dyskinesia, tremor

2. Individual exercise means were not analyzed separately for their specific impact
3. No any qualitative evidence such as NMRI are used after therapy for qualitative measures of outcome.

\section{Evidence with Critical Research Questions}

Movement disorders and their rehabilitation ways needs to be much more evidenced, where a huge gap persists. Through this presentation where a single subject who was regularly treated with Syndopa an exercises, a retrospective 9 years of clinical prognosis and various factors influencing therapy outcome are analyzed with available evidence as below:

1. Does post CABG and PD have an association? Taghipour etal 2011 have evaluated among 112 Iranian subjects post CABG in a one year follow up study have recorded that an improved physical emotional function and general health, but longer term health related quality of life after CABG is worse than that of general population as recorded by (Hanneg Jello et al 2006). As this subject is also a diabetic, worse quality of life than non diabetic post $\mathrm{CABG}$ which was evidenced by (Peric et al 2008)

$>$ Poor participation in cardiac rehab programs, and non compliance with prescribed medications among CAD subjects have poor mental health condition even after CABG (Simchen et al 2001), similar to these reports this subject post CABG has maintained a low physically active life style.

2. Is there a genetic link among subjects with PD decides pattern of progression?

$>$ As the subjects elder sister was diagnosed with PD at the age of 70 years and had a poor prognosis, was bed ridden for 3 years and died at her age of 80 years, hence this hypothetical question arises, but Indian studies support up to $8.5 \%$ cases of familial PD (Biswas et al 2006)

3. Adherence to exercises have a role on PD?

$>$ Adherence to structured exercise program in a one year follow up among 76 Australian stroke subjects have identified several factors 
with increased exercise participation, including high exercise self efficacy, past exercise history, good general health, functional independence, moderate duration activity, program accessibility, convenience , emphasis on social aspects, strong leadership and individually tailored exercise (Anne Tiedmann etal 2012)

$>$ Disease duration and pain are likely to influence negatively for exercise adherence among PD subjects in a six months study with supervised and non supervised exercises (Allen etal 2015)

$>$ As found this study subject with 8 year duration with PD and pain in the knee joints and lumbar sacral junction with an increased falls frequency of recent development could form the basic for his non adherence with exercises and physical activities.

4. Depression, perception and personality do they influence on clinical outcome?

> Behari et al 2001 have among 377 subjects with PD have found male gender, family history of PD, past history of depression up to 10 years, well water drinking of more than 10 years were associated with PD with which this study subject fits full for preponderance to PD

5. Does longer duration being P.D has negative influence?

$>$ In an eight year follow up among 232 patients with PD have established age and longer duration with PD a decline of 3\%was recorded annually in UPDRS score, hoehn and yahr staging (Alexander et al 1986). Habits such as smoking, though this study subject was a non smoker, but interestingly tobacco consumption has a protective effect against PD as supported by (Brown et al 2003 \& Vlaanderen et al 2014). With more than 9 years this subject with P.D is likely have negative prognosis on his QOL as found in his clinical prognosis mentioned earlier.

6. Does socioeconomic status have a say on PD? An increase of age adjusted prevalence rates of PD among the slum population compared with non slum population might indicate a protective effect due to some environmental factor which needs to be probed further has a good economical backup and an urban resident (Tim Crocker 2017), but contrary this subject had an affluent, affordable economic status.

7. Systemic illnesses have any impact on PD?

$>$ This subject being a hypertension and diabetic mellitus on medication for 15 years and being a hypertensive which may accelerate neuro degeneration, as supported by (Dela etal 2002)

Future Recommendations includes larger sample size, combined interdisciplinary approaches and comparing individual exercise variables for efficiency with evidence can be carried out.

\section{Conclusion}

Comprehensive management of a subject with movement disorder is multi factorial requiring pharmacological therapy, physiotherapy but family support individual's perception adherence to exercises, environment etc. this original 8 years follow up of a subject with PD highlightnes various factors influencing prognosis of PD.

\section{References}

1. Tomlinson systematic reviewer, Smitaa Patel statistician, Charmaine Meek research assistant, Clare $\mathrm{P}$ Herd research associate. Physiotherapy intervention in Parkinson's disease: systematic review and meta-analysis. BMJ 2012;345:e5004, PP: 1-14

2. Rascol O, Brooks DJ, Korczyn AD, et al. A five-year study of the incidence of dyskinesia in patients with early Parkinson's disease who were treated with ropinirole or levodopa. 056 Study Group. N Engl J Med. 2000;18:1484-91.

3. Schrag A, Quinn N. Dyskinesias and motor fluctuations in Parkinson's disease: 
a community-based study. Brain. 2000;123(11):2297-2305.

4. Weintraub S. The record of independent living: an informant-completed measure of activities of daily living and behavior in elderly patients with cognitive impairment. Am J Alzheimers Dis Other Dement. 1986; 1:35-9.

5. Andrew Steptoe, Aparna Shankar, Panayotes Demakakos, and Jane Wardle. Social isolation, loneliness, and all-cause mortality in older men and women. Proc Natl Acad Sci U S A.2013 Apr 9; 110(15): 5797-5801.

6. Gage H, Storey L. Rehabilitation for Parkinson's disease: a systematic review of available evidence. Clin Rehab 2004;18:463-82.

7. Kim SD, Allen NE, Canning CG, Fung VS. Postural instability in patients with Parkinson's disease. Epidemiology, pathophysiology and management. CNS Drugs. 2013;27:97-112.

8. Taghipour, $\mathrm{MH}$ Naseri, $\mathrm{R}$ Safiarian, $\mathrm{Y}$ Dadjoo, B Pishgoo, HA Mohebbi, L Daftari Besheli, M Malekzadeh, A Kabir. Quality of Life One Year after Coronary Artery Bypass Graft Surgery. Iranian Red Crescent Medical Journal. Iran Red Crescent Med J 2011; 13(3):171-177

9. Hanne Gjeilo K, Wahba A, Klepstad P, Lydersen S, Stenseth R. Healthrelated quality of life three years after coronary surgery: a comparison with the general population. Scand Cardiovasc J. 2006;40:29-36.

10. Peric V, Borzanovic M, Stolic R, Jovanovic A, Sovtic S, Dimkovic S, Marcetic Z. Predictors of worsening of patients' quality of life six months after coronary artery bypass surgery. J Card Surg. 2008;23:648-54.

11. Simchen E, Naveh I, ZitserGurevich Y, Brown D, Galai N. Is participation in cardiac rehabilitation programs associated with better quality of life and return to work after coronary artery bypass operations? The Israeli CABG Study. Isr Med Assoc J. 2001;3:399-403.

12. Biswas .,Abhijit Biswas \&Neel Das. The Differential Effects of Celebrity and Expert Endorsements on Consumer Risk Perceptions. The Role of Consumer Knowledge, Perceived Congruency, and Product Technology Orientation. Vol. 35, No. 2 (Summer, 2006),Pages 17-31

13. Anne Tiedemann, Catherine Sherrington, Catherine M. Dean, Chris Rissel, Stephen R. Lord, Catherine Kirkham, and Sandra D. O'Rourke. Predictors of Adherence to a Structured Exercise Program and Physical Activity Participation in Community Dwellers after Stroke. Hindawi Publishing Corporation Stroke Research and Treatment Volume 2012, Article ID 136525, 1-8 pages

14. Allen, Jooeun Song, Serene S. Paul, Catherine Sherrington, Susan M. Murray, Sandra D, O'Rourke,. Predictors of Adherence to a Falls Prevention Exercise Program for People with Parkinson's Disease. 2015 International Parkinson and Movement Disorder Society, PP: 395-399

15. Behari M, Srivastava AK, Das RR, Pandey RM. Risk factors of Parkinson's disease in Indian patients. J Neurol Sci. 2001;190: 49-55.

16. Alexander G, Delong M, Strick P. Parallel organization of functionally segregated circuits linking basal ganglia and cortex. Annual Review of Neuroscience. 1986;9:357-381.

17. Brown RA, Ramsey SE, Strong DR, et al. Effects of motivational interviewing on smoking cessation in adolescents with psychiatric disorders. Tobacco Control. 2003;12:3-10.

18. Vlaanderen J, Portengen L, Schuz J, Olsson A, Pesch B, et al. (2014) Effect modification of the association of 
cumulative exposure and cancer risk by intensity of exposure and time since exposure cessation: A flexible method applied to cigarette smoking and lung cancer in the SYNERGY study. Am J Epidemiol 179: 290-298

19. Tim Crocker-Buque, Godwin Mindra, Richard Duncan and Sandra Mounier-Jack. Immunization, urbanization and slums - a systematic review of factors and interventions. Crocker-Buque et al. BMC Public Health (2017) 17:556

20. De La Torre J.C. (2002) Alzheimer disease as a vascular disorder: nosological evidence. Stroke 33: 1152-1162. 